\section{Chief Sensory Nucleus of V}

John E. Mendoza

Department of Psychiatry and Neuroscience, Tulane Medical School and SE Louisiana

Veterans Healthcare System, New Orleans, LA, USA

\section{Synonyms}

Principal sensory nucleus of the trigeminal nerve; Principal sensory nucleus of $\mathrm{V}$

\section{Definition}

Nucleus responsible for proprioceptive feedback from the muscles of facial expression, stereognosis or fine tactual discrimination, and vibratory sensations from the face. Located in the dorsolateral pons just medial to the middle cerebellar peduncle and inferior to the superior cerebellar peduncle, it is the functional equivalent of the nuclei cuneatus and gracilis in the medulla, which mediate similar input from the trunk and extremities. It gives rise to trigeminothalamic fibers, which terminate in the ventral posterior medial nucleus of the thalamus.

\section{Current Knowledge}

Because of its size and density, it is rare for brainstem lesions to be isolated to a single nucleus or pathway. Theoretically, lesions which involve this nucleus might most readily be distinguished on a routine neurological exam by changes (asymmetries) in two-point discrimination on the ipsilateral face. In practice, however, such lesions are likely to involve other brainstem nuclei and pathways, including the adjacent motor nucleus of $\mathrm{V}$, spinal trigeminal tract and/or nucleus, spinal thalamic tracts, lateral portions of the medial lemniscus, and middle cerebellar peduncles resulting in ipsilateral muscle weakness of the jaw muscles, ipsilateral changes in pain and temperature in the face and diminished or abolished corneal reflex, contralateral loss of pain and temperature in the extremities, diminished or loss of proprioception, stereognosis, and vibration in the contralateral extremities (leg > arm), and ipsilateral cerebellar signs.

\section{References and Readings}

Gilman, S., \& Newman, S. W. (2003). Manter and Gatz's essentials of clinical neuroanatomy and neurophysiology. Philadelphia: F.A. Davis.

Wilson-Pauwek, L., Akesson, E. J., Stewart, P. A., \& Spacey, S. D. (2002). Cranial nerves in health and disease. Hamilton: B.C. Decker. 